\title{
Clinical Characteristics, Treatment Outcomes, and its Predictors Among Hospitalized Stroke Patients in Ambo University Referral Hospital, West Ethiopia: A Retrospective Hospital-Based Study
}

This article was published in the following Dove Press journal: Vascular Health and Risk Management

\author{
Diriba Alemayehu Gadisa (iD) \\ Guye Betero Busawa (iD ${ }^{2}$ \\ Esayas Tadesse Gebremariam (iD) \\ Gosaye Mekonen Tefera (iD)' \\ Kefyalew Taye Belete (iD ${ }^{3}$ \\ Getu Melesie Taye' \\ Lemma Bose Meskele' \\ Biruk Mosisa Gudeta' \\ Belayneh Kefale Gelaw (iD ${ }^{4}$ \\ 'Department of Pharmacy, College of \\ Medicine and Health Sciences, Ambo \\ University, Ambo, Ethiopia; ${ }^{2}$ Department \\ of Pharmacy, College of Medicine and \\ Health Sciences, Bule Hora University, \\ Bule Hora, Ethiopia; ${ }^{3}$ Department of \\ Public Health, College of Medicine and \\ Health Science, Ambo University, Ambo, \\ Ethiopia; ${ }^{4}$ Department of Pharmacy, \\ College of Medicine and Health Sciences, \\ Debre Tabor University, Debre Tabor, \\ Ethiopia
}

Background: Stroke is becoming a leading cause of death and adult disability in the developing world.

Objective: The objective of the study was to assess the clinical characteristics, treatment outcomes, and its predictors among stroke patients admitted to Ambo University Referral Hospital (AURH), West Ethiopia.

Methods: All 111 stroke patients hospitalized from April 2016 to May 2019 were included using a hospital-based retrospective study. Socio-demographic profile, past medication history, clinical presentation, drugs prescribed for comorbid condition and stroke treatment, and outcomes data were collected using data abstraction formats and results were summarized using both descriptive and inferential statistics.

Key Findings: According to WHO clinical criteria, 89 (80.2\%) were diagnosed with ischemic stroke, and $20(18.0 \%)$ were diagnosed with hemorrhagic stroke. The majority of the patients $94(84.7 \%)$ had at least one antecedent risk factor in which hypertension accounted for $44.1 \%$. More than half, 69 (62.2\%), of hospitalized stroke patients had good treatment outcomes while $42(37.8 \%)$ had a poor outcome. The overall case-fatality rate was $18(16.2 \%), 12(13.5 \%)$ from ischemic stroke, and $6(30.0 \%)$ from hemorrhagic stroke. Acetyl salicylic acid (ASA) with atorvastatin/simvastatin, $67(73.5 \%)$ in ischemic stroke and $12(60.0 \%)$ in hemorrhagic stroke, was the most frequent medications prescribed for stroke during admission while it was frequently prescribed for $48(53.6 \%)$ of ischemic stroke and $10(50.0 \%)$ of the hemorrhagic stroke on discharge. Overall, ceftriaxone + metronidazole 32 $(28.8 \%)$ was frequently prescribed concomitant medications during admission. The major predictors identified for poor stroke outcomes were substance abuse ( $\mathrm{AOR}=2.839, \mathrm{P}=0.024)$ and have had not received any medication for stroke treatment during admission $(\mathrm{AOR}=12.503, \mathrm{P} \leq 0.001)$.

Conclusion: A significant number of the admitted stroke patients had poor treatment outcome in which the case-fatality rate was relatively high. Substance abuse and having not received medication for stroke were the significant predictors for poor treatment outcome. Hence, a well-established stroke unit setup at AURH is required for accurate diagnosis and management of stroke to overcome stroke-related mortality and/or poor treatment outcome.

Keywords: stroke, predictors, outcomes, Ambo, Ethiopia
Correspondence: Diriba Alemayehu Gadisa Department of Pharmacy, College of Medicine and Health Sciences, Ambo University, P.O. Box 19, Ambo, Ethiopia Tel +251915958190

Email direalexphar@ymail.com
Vascular Health and Risk Management 2020:16 591-604

submit your manuscript

DovePres:

$f$ in - cc) (1) ( 2020 Gadisa et al. This work is published and licensed by Dove Medical Press Limited. The full terms of this license are available at https://www.dovepress.com/terms. work you hereby accept the Terms. Non-commercial uses of the work are permitted without any further permission from Dove Medical Press Limited, provided the work is properly attributed. For permission for commercial use of this work, please see paragraphs 4.2 and 5 of our Terms (https://www.dovepress.com/terms.php). 


\section{Introduction}

According to the World Health Organization (WHO), stroke is a focal or global disturbance of cerebral blood circulation which be either ischemic or hemorrhagic with symptoms lasting 24 hours or longer or leading to death. ${ }^{1}$

Stroke is one of the main causes of death and adult disability in the world, although the burden (high in developing countries) and risk factors vary. For instance, in the United States, 800,000 stroke cases were encountered per year, and among which 128,932 deaths occurred in the year 2011 which gives the case-fatality rate $16.1 \%{ }^{2}$ The study in sub-Saharan Africa reported up to $30 \%$ of the case-fatality rate due to stroke. ${ }^{3}$

Although stroke incidence in high-income countries (HICs) decreased over the decades, the burden is increasing dramatically (more than double) in low- and middleincome countries (LMICs). ${ }^{4}$ Around $70 \%$ of approximately 17 million per annum stroke cases occur in LMICs with limited access to computed tomography (CT) scan before initiation of intravenous tissue plasminogen activator (t-PA) in most patients. ${ }^{5}$

Current guidelines for the management of acute stroke recommend all patients admitted to hospital with suspected acute stroke should receive brain imaging evaluation like computed tomography scanner on arrival to hospital to decide whether the patients need to undergo thrombolysis using tissue plasminogen activator (t-PA) or not. ${ }^{6,7}$ Unfortunately, LMICs face challenges due to a lack of resources, awareness, and technical capacity to accurately diagnose and manage the stroke. ${ }^{5,8}$

The risk factors for stroke are diverse depending on patient outcomes. Hypertension, cardiac disease, and diabetes were commonly reported risk factors of stroke in the world. ${ }^{9-12}$ developed countries, stroke severity, functional disability, high blood pressure, older age, dysphagia, and infections such as pneumonia were reported as the major predictors of poor stroke outcomes. ${ }^{13,14}$ In developing countries, in addition to the above-mentioned factors, stroke symptoms awareness, health-care facilities accessibility, financial problems, support systems at home, being depending on traditional healers, and poor health facility infrastructures were also cited as the major contributing factors for poor outcome of stroke patients. ${ }^{10,15,16}$

The Ethiopian situation is not different from the rest of the world. In 2017, stroke accounted for 39,571 (6.23\%) of total deaths in the country that ranked 6 th. ${ }^{17,18}$ The magnitude of the problem is expected to worsen due to limited stroke treatment resources in the health-care system of the country in which communicable disease is the primary target. However, early detection and treatment of hypertension might be used as primary prevention for stroke in the primary care setting..$^{10,19}$ Overall, although there are studies that reveal the burden of stroke in Ethiopia, there is a lack of objective evidence due to controversial results on the outcome and type of stroke as well as its predictors. ${ }^{20,21}$ Therefore, this study aimed to assess the clinical characteristics, treatment outcomes, and its predictor among stroke patients admitted to Ambo University Referral Hospital (AURH), West Ethiopia.

\section{Methods}

\section{Study Design and Setting}

A retrospective study of patients with stroke admitted to AURH from April 2016 to May 2019 was conducted. AURH is one of the public hospitals found in Ambo town with a population of 76,774 in 2018. ${ }^{22}$ The hospital had more than 250 beds with 4 adult medical outpatient clinics and 1 adult intensive care unit. AURH is used as a teaching center for Ambo University College of Medicine and Health Sciences. Data were extracted from June 1 to June 10, 2019.

\section{Sampling}

Patients who were below 18 years of age and who had incomplete medical records were excluded from the study. Accordingly, all 111 patients with a clinical diagnosis of stroke and admitted to the hospital were included in the study.

\section{Data Collection and Analysis}

The data were collected by two graduate pharmacy students using the data collection sheet. The tool contains questions about socio-demographic characteristics, past medication history, clinical presentation, medication for stroke treatment, and outcomes of admitted stroke patients, and it was adapted after reviewing different kinds of literature. ${ }^{10,16,19}$ The data collection tool was pre-tested on 5 patient charts which were not included in the study. Data collectors were trained for half a day on how to collect the data. Supervision was made by the principal investigators during the data collection process and any inconsistencies were amended on time.

After the data were checked for completeness and consistencies, it was entered and analyzed using SPSS version 25 . Descriptive statistics including frequency mean, percentage, and standard deviations were used to present the result. Bivariate and multivariable backward logistic regressions 
were used to verify the predictors of the poor treatment outcomes. Independent variables with p-value $<0.20$ in bivariate logistic regression were included in the final model, multivariable backward logistic regression, and a p-value of less than 0.05 in the multivariable backward binary logistic regression was considered as statistically significant.

\section{Ethical Approval}

Ethical approval was obtained from the Ethics Review Committee of the Department of Pharmacy; Ambo University with reference number ERB/PHAR/59/01/ 2018. The data collected from the medical records were handled with strong confidentiality and all the collected patient information was stored anonymously. Patient informed consent was not required due to the deidentified data, and that this study was conducted in accordance with the Declaration of Helsinki. ${ }^{23}$

\section{The Standard Protocols for Stroke Diagnosis and Management at AURH}

Even though globally there are numerous protocols for stroke management, ${ }^{6,7,24}$ Ethiopian hospitals, in particular AURH, have not well-established stroke units or stroke teams (ie, no cranial computed tomography (CT scan) or magnetic resonance imaging (MRI) scan, no neurologist) during the study period. Moreover, no official national protocol for acute stroke management during the study period. Even guideline by Ethiopian Medicine, Food and Healthcare Administration and Control Authority (EMFHACA) for general hospitals does not put any recommendation and protocol regarding stroke management in Ethiopia. ${ }^{25}$ Hence, the management and diagnosis of stroke subtype were made by physicians at AURH based on WHO clinical criteria ${ }^{26}$ or based on the management of acute stroke in low-resource settings criteria. ${ }^{5,27}$

\section{Operational Definition}

Undetermined Type of Stroke

A type of stroke neither ischemic nor hemorrhagic not identified.

\section{A Good Treatment Outcome}

If the patient is discharged without any complication secondary to the stroke attack.

\section{Poor Treatment Outcome}

If patients were discharged with any complication or died.

\section{Results}

\section{Socio-Demographic Characteristics}

A total of 111 stroke patient charts were reviewed. Half $(50.5 \%)$ of the patients were females. The mean age of the patients was 63.4 years $(\mathrm{SD} \pm 12.6)$. About $37(33.3 \%)$ of them were substance abusers and $71(64 \%)$ were living in rural areas as shown (see Table 1).

\section{Clinical Characteristics of the Study Subjects}

According to WHO clinical criteria, among 111 patients admitted with stroke, 89 (80.2\%) were diagnosed with ischemic stroke, 20 (18.0\%) were hemorrhagic stroke and the remaining $2(1.8 \%)$ were undetermined. Hemi-paresis was a clinical presentation in $85(76.6 \%)$ patients (ie, $45 \%$ left side and $40 \%$ right side weakness), and only 28 (25.2\%) had a Computed Tomography (CT) scan in which 23 (20.7\%) were from an ischemic stroke while $4(3.6 \%)$ were from hemorrhagic stroke. The majority, 74 (66.7\%) from ischemic stroke and 19 (17.1\%) from hemorrhagic stroke, had at least one comorbidity/antecedent risk factor. Hypertension 39 (35.1\%) and 9 (8.1\%), and atrial fibrillation $15(13.5 \%)$ and $7(6.3 \%)$ were the two most frequently identified comorbidity/antecedent risk factors among patients with ischemic and hemorrhagic stroke, respectively. Overall 24 (21.6\%), 21 (18.9\%) from ischemic stroke, and 7 (6.3\%) from hemorrhagic stroke, patients developed complications like aspiration pneumonia during admission (see Table 2). Thirty-three (29.7\%) from ischemic stroke and $4(3.6 \%)$ from hemorrhagic stroke had a history of substance use. The overall mean time of hospital arrival since the onset of the symptoms and hospitalization was $58.15( \pm 1.6)$ hours and $6.02( \pm 3.24)$ days, respectively. In general, no variables (ie, clinical characteristics) have shown significant association with specific types of stroke ( $\mathrm{P} \geq 0.161)$ (Table 2).

\section{Pre-Stroke and Concurrent Medication History of the Study Participants for Antecedent Risk Factors and \\ Complications}

Enalapril+Metoprolol/Atenolol, 18 (20.2\%) in ischemic stroke and $3(15.0 \%)$ in hemorrhagic stroke, had been frequently prescribed with pre-stroke medications for pre-existed antecedent risk factors like hypertension and/ or heart disease. However, during the admission/hospitalization, ceftriaxone + metronidazole, $23(25.8 \%)$ in ischemic stroke and $9(45.0 \%)$ in hemorrhagic stroke, 
Table I Socio-Demographic Characteristics of Stroke Patients at Ambo University Referral Hospital from March 2016 to May 2019 GC Ambo, Ethiopia ( $\mathrm{N}=\mathrm{III})$

\begin{tabular}{|c|c|c|}
\hline \multicolumn{2}{|l|}{ Variables } & \multirow{2}{*}{$\begin{array}{l}\begin{array}{l}\text { Frequency } \\
(\%)\end{array} \\
25(22.5) \\
86(77.5) \\
63.4 \pm 12.18\end{array}$} \\
\hline Age & $\begin{array}{l}\leq 50 \\
>50 \\
\text { Mean age }\end{array}$ & \\
\hline Sex & $\begin{array}{l}\text { Male } \\
\text { Female }\end{array}$ & $\begin{array}{l}55(49.5) \\
56(50.5)\end{array}$ \\
\hline Marital status & $\begin{array}{l}\text { Single } \\
\text { Married } \\
\text { Divorced }\end{array}$ & $\begin{array}{l}5(4.5) \\
97(87.4) \\
9(8.1)\end{array}$ \\
\hline Occupation & $\begin{array}{l}\text { Farmers } \\
\text { Housewife } \\
\text { Civil servant } \\
\text { Others }^{\neq}\end{array}$ & $\begin{array}{l}44(39.6) \\
43(38.7) \\
14(12.6) \\
10(9.0)\end{array}$ \\
\hline $\begin{array}{l}\text { Educational } \\
\text { status }\end{array}$ & $\begin{array}{l}\text { Unable to read and write } \\
\text { Able to read and write, informal } \\
\text { education } \\
\text { Above secondary school }\end{array}$ & $\begin{array}{l}68(61.3) \\
26(23.4) \\
17(15.3)\end{array}$ \\
\hline Religion & $\begin{array}{l}\text { Orthodox } \\
\text { Muslim } \\
\text { Protestant }\end{array}$ & $\begin{array}{l}30(27.0) \\
22(19.8) \\
59(53.2)\end{array}$ \\
\hline Residence & $\begin{array}{l}\text { Urban } \\
\text { Rural }\end{array}$ & $\begin{array}{l}40(36) \\
71(64)\end{array}$ \\
\hline Ethnicity & $\begin{array}{l}\text { Amhara } \\
\text { Oromo }\end{array}$ & $\begin{array}{l}14(12.6) \\
97(87.4)\end{array}$ \\
\hline
\end{tabular}

Note: ${ }^{\ddagger}$ Indicates, Students, unemployed, merchant.

was frequently used as a concurrent medication for stroke complication like aspiration pneumonia. Overall, 57 (51.4\%) of the study participants, 43 (48.3\%) from ischemic, and $12(60.0 \%)$ from hemorrhagic stroke did not have pre-stroke medication (past medication history). Among 23 (20.7\%) of study participants who did not receive concurrent medication during admission for their comorbidity, $21(23.6 \%)$ were from ischemic stroke (see Table 3).

\section{Medications Used for Stroke Treatment by the Study Subjects}

Sixty-seven (75.3\%) patients from ischemic stroke and 12 $(60.0 \%)$ patients from hemorrhagic stroke were frequently prescribed with aspirin+atorvastatin/simvastatin during admission. Likely, 48 (53.6\%) patients from ischemic stroke and $10(50.0 \%)$ patients from hemorrhagic stroke were frequently discharged with aspirin+atorvastatin/simvastatin. However, no stroke medications were prescribed for $14(15.7 \%)$ and $23(25.8 \%)$ patients with ischemic stroke, and each $7(35.0 \%)$ patients with hemorrhagic during admission and discharge, respectively. None of the patients had received intravenous t-PA during hospital admission because of the unavailability of the medications and required facilities (see Table 4).

\section{Stroke Treatment Outcome Among the Study Participants}

Overall $69(62.2 \%)$ of patients, $58(65.2 \%)$ from ischemic stroke and $10(50.0 \%)$ from hemorrhagic stroke, had good treatment outcomes (ie, discharged without any complication) and the rest 42 (37.8\%) patients, 31 (34.8\%) from ischemic and $10(50.0 \%)$ from hemorrhagic stroke had poor treatment outcome (see Figure 1). The overall in-hospital case-fatality rate was $18(16.2 \%), 12(13.5 \%)$ from ischemic stroke, and 6 (30.0\%) from hemorrhagic stroke. However, among 93 patients discharged alive, 24 (21.6\%) were discharged with a neurological deficit (see Figure 2). Among 18 patients dead, aspiration pneumonia $12(66.7 \%)$ and increased intracranial pressure $3(16.7 \%)$ were the most frequently documented cause of death secondary to stroke (see Figure 3).

\section{Predictors of Stroke Treatment Outcome Among the Study Participants}

On bivariate logistic regression substance abuse ( $\mathrm{COR}=2.341, \mathrm{P}=0.040)$, being civil servant from occupational status $(\mathrm{COR}=7.246, \mathrm{P}=0.004)$, having severe stroke type $(\mathrm{COR}=4.533, \mathrm{P}=0.001)$, having complication during admission $(\mathrm{COR}=2.393, \mathrm{P}=0.05)$ and having no any medication for stroke treatment during admission $(\mathrm{COR}=11.05, \mathrm{P} \leq 0.001)$ were the independent factors that increase the likely hood of poor treatment outcome. On contrary, being illiterate or having informal education $(\mathrm{COR}=0.186-0.221, \mathrm{P} \leq 0.025)$ and being a rural resident ( $\mathrm{COR}=0.449, \mathrm{P}=0.049)$ were the significant independent predictors that decrease the likelihood of poor treatment outcome at $\mathrm{P}=0.05$. However, the multivariable backward logistic regression analysis revealed that substance abuse $(\mathrm{AOR}=2.839, \mathrm{P}=0.024)$ and have had not received any medication for stroke treatment during admission (AOR=12.503, $\mathrm{P} \leq 0.001)$ were the two independent factors that increased the likely hood of poor stroke treatment outcome among our study participants (see Table 5). 


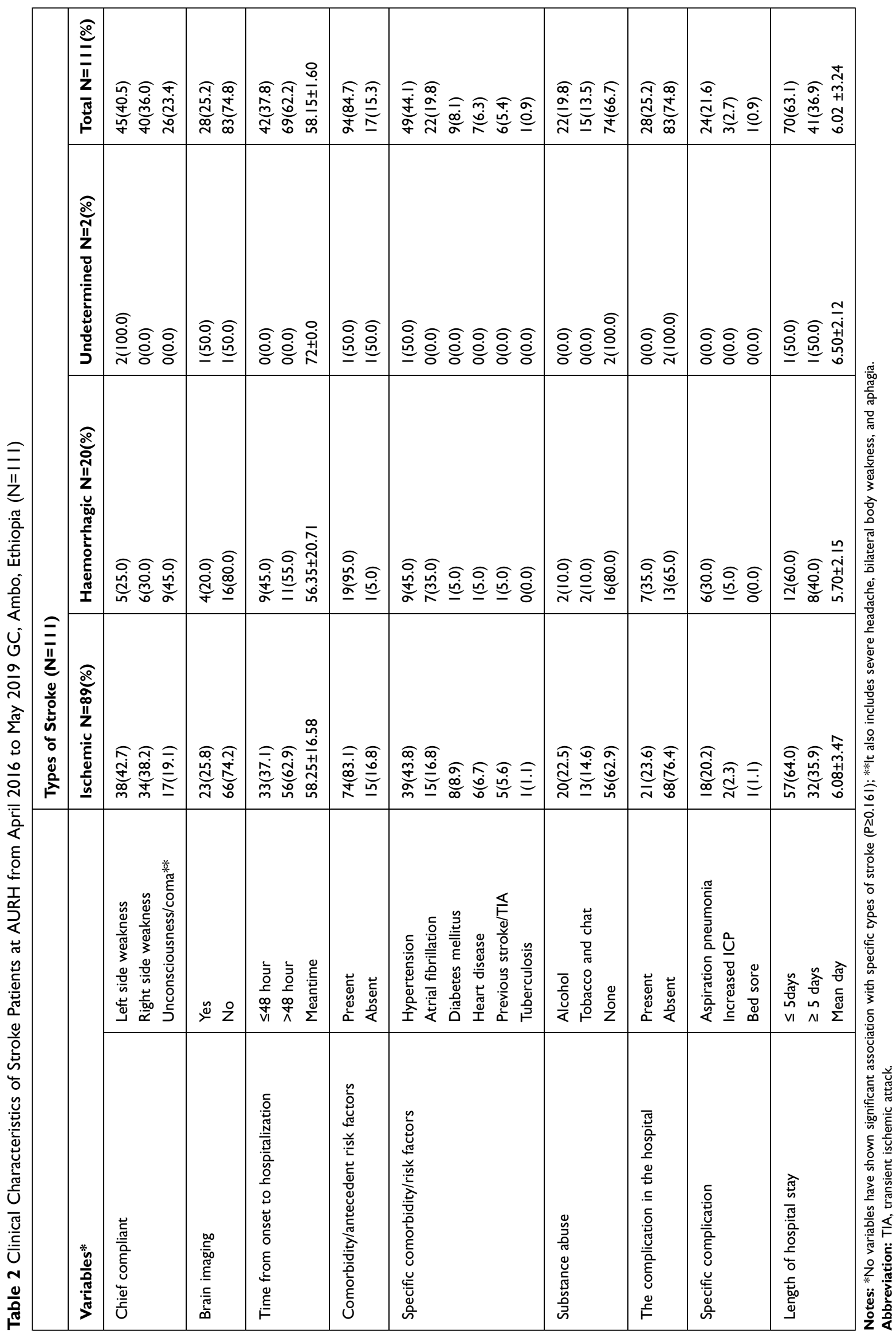


Table 3 Pre-Stroke and Concurrent Medication History of Hospitalized Stroke Patients for Antecedent Risk Factors at AURH, from March 2016 to May 2019 GC, Ambo, Ethiopia (N=III)

\begin{tabular}{|c|c|c|c|c|}
\hline & \multicolumn{4}{|c|}{ Types of Stroke $(\mathrm{N}=\mathrm{I} I \mathrm{I})$} \\
\hline & $\begin{array}{l}\text { Ischemic } \mathbf{N}=89 \\
(\%)\end{array}$ & $\begin{array}{l}\text { Haemorrhagic } \mathbf{N}=\mathbf{2 0} \\
\text { (\%) }\end{array}$ & $\begin{array}{l}\text { Undetermined } \mathbf{N}=\mathbf{2} \\
(\%)\end{array}$ & $\begin{array}{l}\text { Total } \mathbf{N}=\mid \text { I I } \\
(\%)\end{array}$ \\
\hline \multicolumn{5}{|l|}{ Pre-stroke medication (past medication history) } \\
\hline Enalapril+Metoprolol/Atenolol & $18(20.2)$ & $3(15.0)$ & $0(0)$ & $21(18.9)$ \\
\hline Enalapril + Hct/Furosemide & $10(11.2)$ & $I(5.0)$ & $0(0)$ & $11(9.9)$ \\
\hline Digoxin + Metoprolol/Atenolol & $5(5.6)$ & $2(10.0)$ & $0(0)$ & $7(6.3)$ \\
\hline Digoxin + Enalapril \pm Atenolol & $4(4.5)$ & $I(5.0)$ & $0(0)$ & $5(4.5)$ \\
\hline Metformin/NPH insulin & $8(8.9)$ & $\mathrm{I}(5.0)$ & $0(0)$ & $9(8.1)$ \\
\hline RHZE* & $\mathrm{I}(\mathrm{I} . \mathrm{I})$ & $0(0.0)$ & $0(0)$ & $\mathrm{I}(0.9)$ \\
\hline No medication & $43(48.3)$ & $12(60.0)$ & $2(100.0)$ & $57(51.4)$ \\
\hline \multicolumn{5}{|c|}{ Concurrent medications during admission/hospitalization } \\
\hline Ceftriaxone + Metronidazole & $23(25.8)$ & $9(45.0)$ & $\mathrm{I}(50.0)$ & $33(29.7)$ \\
\hline Ceftriaxone + Metronidazole+Mannitol & $2(2.3)$ & $3(15.0)$ & $0(0.0)$ & $5(4.5)$ \\
\hline Ceftriaxone + Azithromycin & $2(2.3)$ & $0(0.0)$ & $0(0.0)$ & $2(1.8)$ \\
\hline Amlodipine + Captopril/Enalapril & $8(8.9)$ & $\mathrm{I}(5.0)$ & $0(0.0)$ & $9(8.1)$ \\
\hline Amlodipine+ Captopril/Enalapril+Mannitol & $3(3.4)$ & $0(0.0)$ & $0(0.0)$ & $3(2.7)$ \\
\hline Enalapril only & $10(\mid 1.2)$ & $2(10.0)$ & $0(0.0)$ & $12(10.8)$ \\
\hline Digoxin+Metoprolol+Enalapril/Captopril & $2(2.3)$ & $2(10.0)$ & $0(0.0)$ & $4(3.6)$ \\
\hline Digoxin+Metoprolol+Enalapril/Captopril+ & $\mathrm{I}(\mathrm{I} . \mathrm{I})$ & $0(0.0)$ & $0(0.0)$ & $\mathrm{I}(0.9)$ \\
\hline \multicolumn{5}{|l|}{ Mannitol } \\
\hline Digoxin + Hct/Furosemide & $3(3.4)$ & $0(0.0)$ & $0(0.0)$ & $3(2.7)$ \\
\hline Digoxin only & $4(4.5)$ & $0(0.0)$ & $0(0.0)$ & $4(3.6)$ \\
\hline Atenolol only & $\mathrm{I}(\mathrm{I} . \mathrm{I})$ & $0(0.0)$ & $0(0.0)$ & $\mathrm{I}(0.9)$ \\
\hline Metformin \pm Glibenclamide & $7(7.9)$ & $\mathrm{I}(5.0)$ & $0(0.0)$ & $8(7.2)$ \\
\hline Mannitol only & $2(2.3)$ & $I(5.0)$ & $0(0.0)$ & $3(2.7)$ \\
\hline No medication prescribed & $21(23.6)$ & $2(10.0)$ & $I(50.0)$ & $23(20.7)$ \\
\hline
\end{tabular}

Abbreviations: *RHZE, rifampicin/isoniazid/pyrazinamide/ethambutol for TB treatment; NPH, neutral protamine Hagedorn; Hct, hydrochlorothiazide.

Table 4 Medications Used for Stroke Treatment During Admission and on Discharge by Study Participants at AURH from April 2016 to May 2019 GC, Ambo, Ethiopia ( $\mathrm{N}=11 \mathrm{I})$

\begin{tabular}{|c|c|c|c|c|}
\hline & \multicolumn{4}{|c|}{ Types of Stroke $(\mathrm{N}=|\mathrm{I}| \mathrm{)})$} \\
\hline & Ischemic* $\mathbf{N}=\mathbf{8 9}(\%)$ & Haemorrhagic $\mathbf{N}=\mathbf{2 0}(\%)$ & Undetermined $\mathbf{N}=\mathbf{2}(\%)$ & Total $\mathbf{N}=$ I I I (\%) \\
\hline \multicolumn{5}{|c|}{ Medications used for stroke treatment during admission } \\
\hline Aspirin+Atorvastatin/Simvastatin & $67(75.3)$ & $12(60.0)$ & $2(100.0)$ & $8 I(73.0)$ \\
\hline Aspirin alone & $I(I . I)$ & $0(0.0)$ & $0(0.0)$ & $\mathrm{I}(0.9)$ \\
\hline Aspirin+Atorvastatin +Clopidogrel & $\mathrm{I}(\mathrm{I} . \mathrm{I})$ & $0(0.0)$ & $0(0.0)$ & $\mathrm{I}(0.9)$ \\
\hline Aspirin+Atorvastatin +Warfarin & $6(6.7)$ & $I(5.0)$ & $0(0.0)$ & $7(6.3)$ \\
\hline No medication prescribed & $14(15.7)$ & $7(35.0)$ & $0(0.0)$ & $21(18.9)$ \\
\hline \multicolumn{5}{|c|}{ Medications used for stroke treatment on discharge } \\
\hline Aspirin+Atorvastatin/Simvastatin & $48(53.6)$ & $10(50.0)$ & $2(100)$ & $60(54.1)$ \\
\hline Aspirin+Atorvastatin +Clopidogrel & $\mathrm{I}(\mathrm{I} . \mathrm{I})$ & $0(0.0)$ & $0(0.0)$ & $\mathrm{I}(0.9)$ \\
\hline Aspirin+Atorvastatin +Warfarin & $7(7.9)$ & $\mathrm{I}(5.0)$ & $0(0.0)$ & $8(7.2)$ \\
\hline Warfarin + Atorvastatin & $10(11.2)$ & $2(10.0)$ & $0(0.0)$ & $12(10.8)$ \\
\hline No medication prescribed/dead & $23(25.8)$ & $7(35.0)$ & $0(0.0)$ & $30(27.0)$ \\
\hline
\end{tabular}

Note: *None of the patients received intravenous t-PA because of the unavailability of the drug and required facilities. 


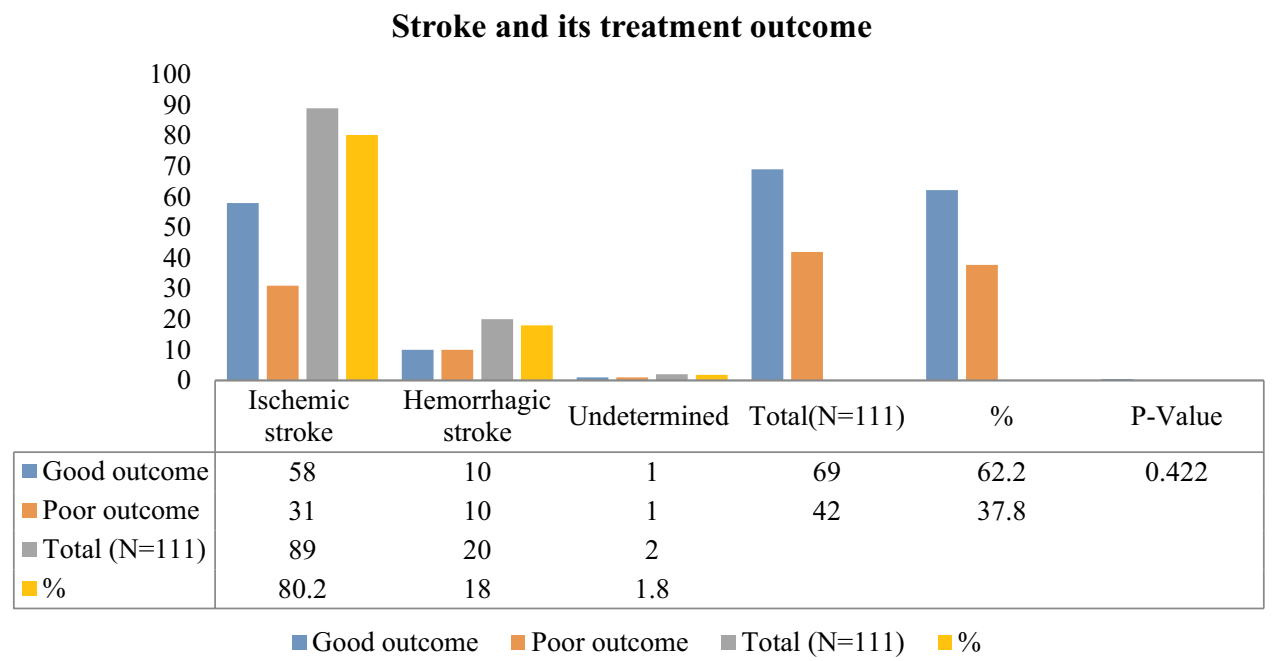

Figure I Stroke type and its treatment outcome among admitted patients with stroke at AURH, from March 2016 to May 2019 GC, Ambo, Ethiopia.

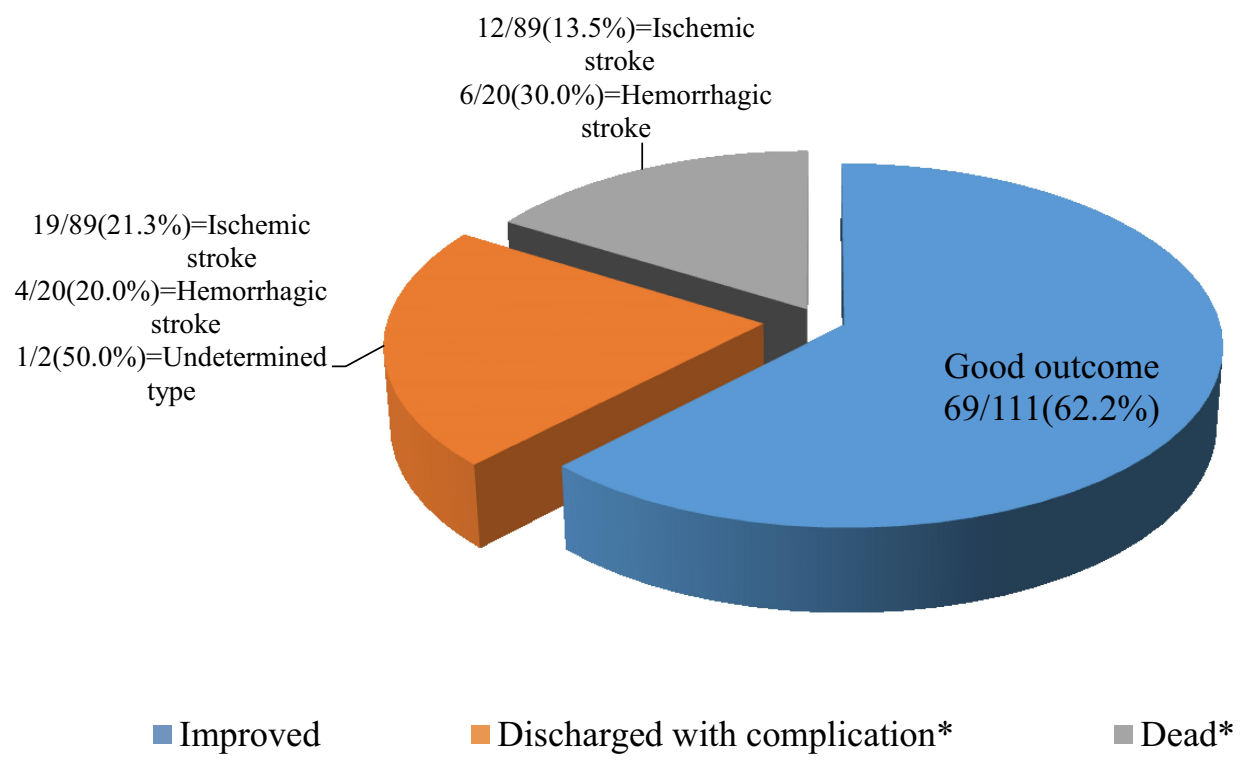

Figure 2 Pattern of treatment outcome among the patients admitted with stroke at AURH, from March 2016 to May 2019 GC, Ambo, Ethiopia.

Note: *Poor outcome.

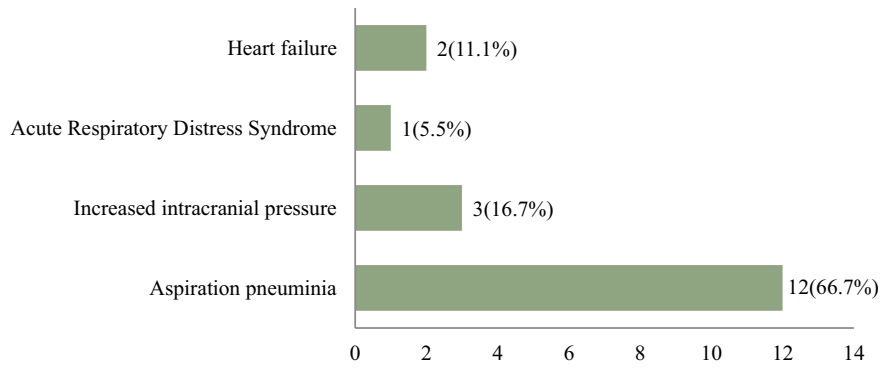

Figure 3 The reason of death secondary to stroke among the patients admitted with stroke at AURH, from March 2016 to May 2019 GC, Ambo, Ethiopia, N=I8. 
There was no association between treatment outcome and types of stroke $\left(\mathrm{X}^{2}\right.$ value=1.726, $\left.\mathrm{P}=0.422\right)$ (see Figure 1 ).

\section{Discussion}

A stroke is the brain equivalent of a heart attack. ${ }^{26}$ It is one of the most common causes of morbidity and mortality worldwide and is a prominent cause of death, disability, and dementia in sub-Saharan Africa including Ethiopia. ${ }^{16,21,28}$

In this study, according to WHO clinical criteria, ischemic stroke was found the most common type of stroke accounting for $80.1 \%$ of the cases, which is much higher than other studies, conducted in different hospitals in Ethiopia where the proportion of Ischemic stroke ranges from $31.5 \%$ to $69.4 \% .^{12,19,20,29-32}$ However, this finding was contrary to other studies in the country where hemorrhagic stroke was the most prevalent among stroke patients. ${ }^{10,28}$ This could be due to differences in the prevalence of cardiovascular risk, where the previous study reported high hypertension prevalence. Hemiparesis leftsided 45 (40.5\%) and right-sided 40 (36\%), was the common chief complaint reported by our study participants on arrival to the hospital which is higher (36-39.7\% and $21.9-29.1 \%$, respectively) than the study reported by Temesgen et $\mathrm{al}^{20}$ and Fikadu et $\mathrm{al}^{21}$ The other study done in North West Ethiopia reported $81.1 \%$ of patients with stroke who sought medical treatment had hemiparesis or hemiplegia. ${ }^{32}$

The present study also showed that 94 (84.7\%) of patients had at least one comorbid condition/antecedent risk factors, ischemic stroke $74(83.1 \%)$ and hemorrhagic stroke 19 (95.0\%), before admission for stroke. Hypertension was the most frequent, ischemic stroke 39 (44.1\%) and hemorrhagic stroke 9 (45.0\%), antecedent risk factor identified. This finding is consistent with the findings from other institutions in Ethiopia. ${ }^{12,19,32,33} \mathrm{~A}$ pooled results of studies published from 2015 to 2019 in Ethiopia indicated that the overall magnitude of hypertension, diabetes mellitus, and atrial fibrillation among stroke patients were $47 \%, 8 \%$, and $10 \%$, respectively. ${ }^{34}$ Similar patterns were also observed in our study, hypertension 49 (44.1\%), atrial fibrillation 22 (19.8), and diabetes mellitus $9(8.1 \%)$. Reports from Gambia and Cameroon ${ }^{35,36}$ indicated that there was poor control of risk factors and poor awareness of the significance of stroke symptoms by patients in developing countries. It is recommended that primary prevention strategies targeting the most common risk factors for stroke in this population including hypertension should be implemented to reduce the problem. ${ }^{32}$ Hence, overall in this study, Enalapril +Metoprolol/Atenolol $21 \quad(18.9 \%)$ and Enalapril +Hydrochlorothiazide (Hct)/Furosemide 11 (9.9\%) had been frequently prescribed for pre-existed antecedent risk factors like hypertension and/or heart disease. On other hand, the study in Nekemte referral hospital, West Ethiopia, found that nifedipine alone $70(19.2 \%)$ was the most frequent pre-stroke medication prescribed. ${ }^{21}$

The finding of the present study revealed that 81 (73\%) of patients were prescribed aspirin with statins (ie, atorvastatin/simvastatin) during hospitalization; this finding was similar to what was found in Shashemene, Ethiopia. $^{20}$ However, aspirin alone was the most prescribed 148 (40.7\%) medication for stroke during admission at Nekemte hospital, West Ethiopia. ${ }^{21}$ In the current study, clopidogrel was infrequently used due to unavailability. Likewise, other previous studies in different hospitals in Ethiopia also reported infrequent use of clopidogrel. $^{20,21}$ At present, literature revealed that the wider and more appropriate use of aspirin and statins will reduce premature stroke and other cardiovascular disease-related death. ${ }^{37}$

An unexpected finding from our study is that a high percentage of hemorrhagic patients with stroke $12(60.0 \%)$ received an aspirin-containing regimen during admission. And, a study indicated that aspirin would increase the risk of bleeding that might lead to hematoma enlargement, poorer outcomes, and increased risk of morbidity and mortality among hemorrhagic patients with stroke. ${ }^{8}$ However, in resource-constrained environments, where barriers to timely CT scans delay clinical decision-making, physicians may balance the risks of aspirin administration against potential complications, ${ }^{27}$ as different findings reported that in-hospital aspirin use was associated with decreased mortality and a decrease rate of in-hospital stroke recurrence among patients with hemorrhagic stroke. $^{32,35}$

Standard care in high-income countries is that all admitted patients with suspected acute stroke should receive a brain scan (eg, CT) to make appropriate stroke management decisions, ${ }^{27}$ and thrombolysis using intravenous t-PA should be administered to eligible patients within 4.5 hours of hospital admission. ${ }^{7}$

Treatment for acute stroke in developing countries is generally symptomatic; thrombolytic and neuroprotective drugs are the exception rather than the rule. ${ }^{4}$ Surprisingly, in the current study, only $1(0.9 \%)$ of patients reached the 


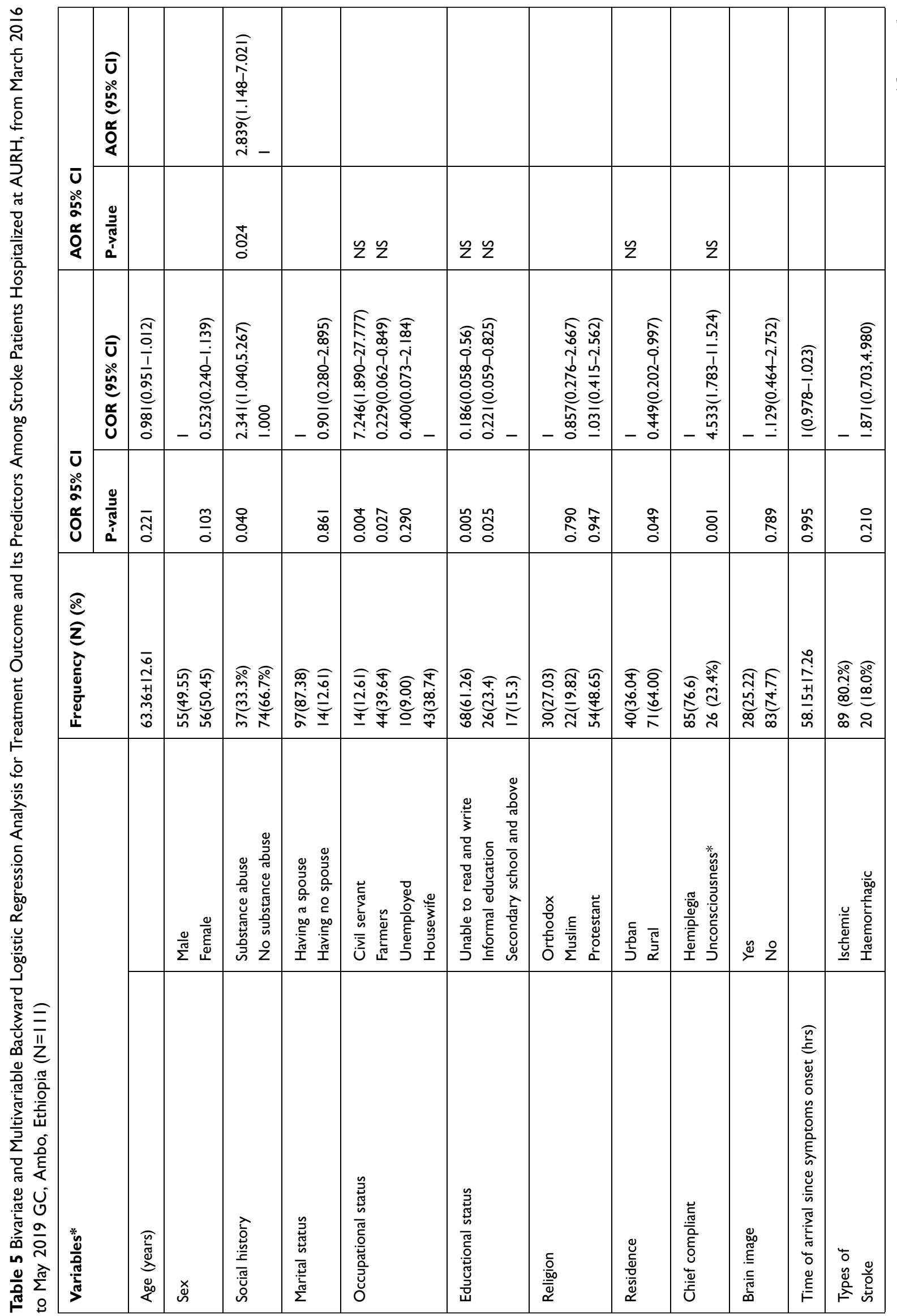




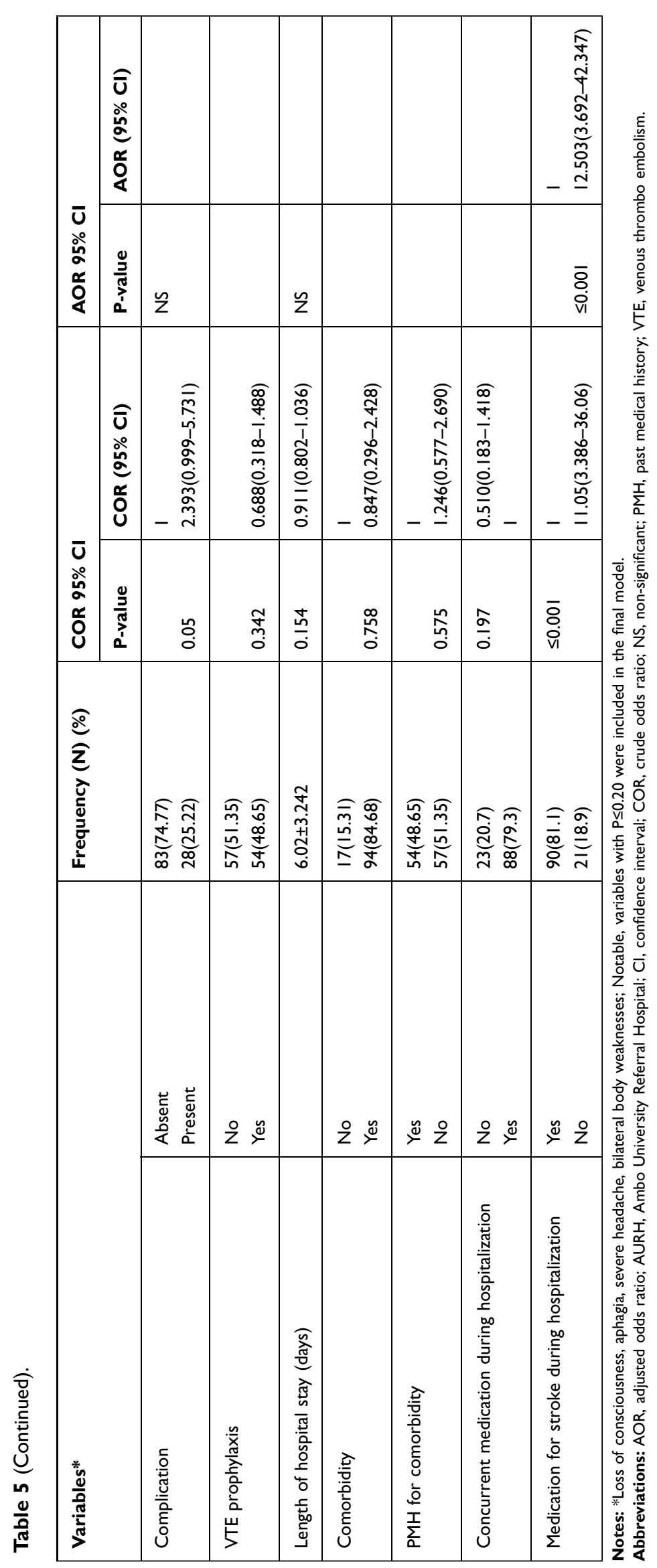


hospital within the first 4.5 hours since symptom onset, and only $28(25.2 \%)$ of patients received a CT scan. In addition to an unavailability of intraveneous t-PA and required facility for thrombolysis like CT scan at AURH, almost all of our patients (99.1\%) did not arrive to the hospital within the required 4.5 hours of symptom onset for thrombolysis. Therefore, all of our patients with ischemic stroke did not undergone thrombolysis. Hence, a country like Ethiopia with financial constraints and lack of infrastructure could not adhere to the standard stroke care established in high-income countries, highlighting the need to establish best practices for stroke care in low- and middle-income countries. ${ }^{32}$

There is no specific treatment, rather than supportive, for patients with hemorrhagic stroke, unlike ischemic stroke. ${ }^{6,7,24}$ Conversely, 7 (35.0\%) of the current study participants with hemorrhagic stroke did not receive specific stroke medication. However, 14 (15.7\%) of patients with ischemic stroke did not receive any stroke medication during their admission. This might be due to physicians unable to accurately diagnose, as the treatments were different for both, the type of stroke the patients suffered from since there was no CT scan and trained human resources in AURH during the study period. The physicians usually withhold aspirin administration particularly if there is a progression of focal neurologic deficits and/or disturbance of consciousness. ${ }^{26}$ Unfortunately, in the current study, those study participants that had not received any stroke medication during admission had at least 12 times odds $(\mathrm{AOR}=12.503, \mathrm{P} \leq 0.001)$ of having poor treatment outcome than their counterparts.

Among patients who developed complications at AURH ( $n=28), 24$ of them suffered from aspiration pneumonia, the other 3 developed increased intracranial pressure, and 1 patient suffered from bedsore. In our setup, ceftriaxone + metronidazole was the most frequent (28.8\%) concomitant medication prescribed for complications that happened during admission. However, Fikadu et $\mathrm{al}^{21}$ found that ceftriaxone + metronidazole + mannitol $92(25.3 \%)$ was the most frequent concurrent medication prescribed for complications in West Ethiopia.

In this study, the overall in-hospital case-fatality rate of stroke was found to be $18(16.2 \%), 12(13.5 \%)$ from ischemic stroke, and $6(30.0 \%)$ from hemorrhagic stroke, which is higher than the case-fatality rate reported from Bahir Dar and Mekelle, Northern Ethiopia (11\% and $14.9 \%$, respectively). ${ }^{29,31}$ However, our finding is slightly lower than what was found in Tikur Anbessa hospital,
Addis Ababa, Ethiopia, and Kenya where the overall mortality rate was $19.2 \%$ and $21.6 \%$, respectively. ${ }^{19,38}$ It is also much lower compared to the case-fatality rates of St. Paul Hospital, Addis Ababa, Ethiopia, 30.1\%, and in the Gambia, 41\%. ${ }^{28,35}$ Moreover, our finding approaches the national stroke-related in-hospital death which was $18 \%{ }^{34}$ The inconsistent results from different hospitals in Ethiopia and abroad might be due to the methodological differences where retrospective study design is considered in this study. However, to understand the outcome of acute stroke, prospective follow-up studies are mandatory. ${ }^{11,29}$ Acute stroke case-fatality rates can be used as one of the quality indicators for acute stroke treatment in the hospital. It is recommended that a designated referral stroke center should be established in the western part of Ethiopia.

Our study also found that nearly two-thirds of 69 (62.2\%) of the stroke patients had good treatment outcomes (ie, discharged without any complications). This finding is similar to other studies conducted in South East and North West Ethiopia where 54 0.8\% and 59.2\% of the patients, respectively, had good treatment outcomes. ${ }^{20,39}$ However, in contrast to our findings, 42 $(37.8 \%)$, a study by Deljavan et $\mathrm{al}^{11}$ reported that $69.2 \%$ of the stroke patients had poor treatment outcomes. The disparity is likely to be due to differences in infrastructure, services, and health professional experiences among the health facilities. Though half of our patients (50.0\%) with hemorrhagic stroke had poor treatment outcomes, we did not find an association between stroke types and treatment outcome $\left(\mathrm{X}^{2}=0.422, \mathrm{P}=0.42\right)$.

The overall mean duration of hospital stay in this study was $6.02 \pm 3.24$ days which is shorter than the study reported from Northern Ethiopia, where the mean length of hospital stay of stroke patients was 11-13 days. ${ }^{39}$ The long duration of time may be due to the severity of symptoms on admission that might be related to poor accessibility to hospital care for less-severe stroke cases and/or the use of anticoagulants like warfarin or DVT prophylaxis during admission. ${ }^{40}$

Our finding revealed that the median (mean) time for hospital arrival from the onset of symptoms was 60 (58) hours. The arrival of the present study participants to the hospital was delayed by far when compared with the other studies in different parts of Ethiopia where it ranges from 7 to 24 hours. $^{20,21,28,31}$ However, inconsistent with our study, one study ${ }^{40}$ in North West Ethiopia reported 60 hours for median time for onset of stroke symptoms to hospital arrival. 
It is recommended that the patients should be admitted within the first golden 3 hours since the onset of stroke symptoms for better treatment outcome. ${ }^{6,7,24}$ However, only $1(0.9 \%)$ patient arrived within 3 hours of onset of the symptoms, and the majority of the patients (62.2\%) arrived after 48 hours of the onset of the symptoms (data not shown). These might be due to the majority (64\%) of the patients were rural residents and the long meantime of hospital admission may also be due to reduced awareness of people about the advantage of early hospital admission and poor health-care infrastructure in a rural area. Access to health care limited in Ethiopia due to different factors such as delayed referrals, lack of ambulance services; proximity to health-care facilities; lack of the specialized health-care center; financial strain and support systems at home, and reliance on natural healers; these affect a patient's ability to reach the health-care facility and to get appropriate care. ${ }^{10,41}$ Hence, much work should be done to improve knowledge and attitude towards stroke treatment and the importance of early admission. However, despite the long delay in symptom onset-to-admission time, it did not show a statistically significant association with poor treatment outcome in our case.

Upon multivariable backward logistic regression, those study participants with a history of substance abuse had almost three times higher $(\mathrm{AOR}=2.839$, $\mathrm{P}=0.024$ ) probability of poor treatment outcome than those who had not. This finding was in line with the studies in northern Ethiopia ${ }^{29,42}$ that reported the significant association of social history with poor treatment outcome. Besides, those patients who did not receive stroke medication during admission had at least twelve times higher likely hood of poor treatment outcomes than their counterparts $(\mathrm{AOR}=12.503, \mathrm{P} \leq 0.001)$. Our findings were contrary to a study by Temesgen et $\mathrm{al}^{20}$ in which any variable was not significantly associated with the treatment outcomes and the other study in West Ethiopia where educational status and length of hospital stay predict poor outcome. ${ }^{21}$ This could be due to sampling size insufficiency to predict significant association in the previous study and difference in the socio-demographic characteristic of study areas. However, other studies conducted in Iran, Switzerland, and Canada reported older age had a significant association with poor treatment outcomes ${ }^{11,43,44}$ contrary to our findings and other studies in Ethiopia. ${ }^{16,28}$

\section{Limitation of the Study}

There was no standard work-out for diagnosis (ie, no CT scan/MRI; no sonography/transcranial doppler to identify whether the cause was from the cardiac or neurovascular origin) and management of stroke at AURH. Hence, diagnosis and management of the stroke were made based on WHO clinical criteria and stroke management guidelines in a resource-limited setting. Unfortunately, this might lead to inaccurate diagnosis and management of ischemic and hemorrhagic stroke. Also, in a place where there is no CT scan, it is difficult to differentiate thalamic hemorrhage from thalamic infarction. Moreover, there was no specialized neurological department at AURH (ie, no neurologist, no stroke unit/team). National Institute of Health Stroke Severity (NIHSS) score was not recorded on patients folder that was not able us to grade the severity of the stroke during the study. Moreover, the study included those admitted to the hospital only, we cannot rule out potential selection bias (referral bias) for those who died out of the hospital. Finally, due to the retrospective nature of the study, our data did not capture patients who experienced a transient ischemic attack whose symptoms may resolve by themselves.

\section{Conclusion and Recommendation}

A significant number of the admitted stroke patients had poor treatment outcome in which the in-hospital casefatality rate was relatively high. History of substance abuse and having had not received stroke medications during admission were the significant predictors for poor treatment outcomes. Hence, a well-established stroke unit setup at AURH is required for accurate diagnosis and management of stroke. Finally, adherence to current standard stroke care protocol is necessary to overcome strokerelated mortality and/or poor treatment outcome.

\section{Data Sharing Statement}

The datasets generated and analyzed during the current study are available from the corresponding author on a reasonable request.

\section{Author Contributions}

All authors contributed to data analysis, drafting or revising the article, have agreed on the journal to which the article be submitted, gave final approval of the version to be published, and agree to be accountable for all aspects of the work. 


\section{Funding}

No fund is received from any funding organizations.

\section{Disclosure}

The authors report no conflicts of interest in this work.

\section{References}

1. WHO MONICA Project Principal Investigators. The world health organization monica project (monitoring trends and determinants in cardiovascular disease): a major international collaboration. $J$ Clin Epidemiol. 1988;41(2):105-114. doi:10.1016/0895-4356(88)90084-4

2. Boan AD, Feng WW, Ovbiagele B, et al. Persistent racial disparity in stroke hospitalization and economic impact in young adults in the buckle of stroke belt. Stroke. 2014;45:1932-1938. doi:10.1161/ STROKEAHA.114.004853

3. Owolabi MO, Akarolo-Anthony S, Akinyemi R, et al. The burden of stroke in Africa: a glance at the present and a glimpse into the future. Cardiovasc J Afr. 2015;26(2):S27-S38. doi:10.5830/CVJA-2015-038

4. Fekadu G, Chelkeba L, Melaku T, et al. Management protocols and encountered complications among stroke patients admitted to stroke unit of Jimma university medical center, Southwest Ethiopia: prospective observational study. Ann Med Surg. 2019;48:135-143. doi:10.1016/j.amsu.2019.11.003

5. Berkowitza AL. Managing acute stroke in low-resource settings. Bull World Heal Organ. 2016;94:554-556. doi:10.2471/BLT.15.162610

6. Iii JCH, Greenberg SM, Cushman M, Fung GL, Mitchell PH, Scott PA. AHA/ASA guideline for the management of spontaneous intracerebral hemorrhage: a guideline for healthcare professionals from the American Heart Association/American Stroke Association. Stroke. 2015;46:2032-2060. doi:10.1161/STR.0000000000000069

7. Powers WJ, Rabinstein AA, Ackerson T, et al. AHA/ASA guideline for the early management of patients with acute ischemic stroke: 2019 update to the 2018 guidelines for the early management of acute ischemic stroke: a guideline for healthcare professionals from the American Heart Association/America. Stroke. 2019;50:e344-418. doi:10.1161/STR.0000000000000211

8. Behrouz R, Miller CM. Aspirin and intracerebral hemorrhage: where are we now? Neurol Clin Pract. 2015;5:11-16. doi:10.1212/ CPJ.0000000000000089

9. Sarfo FS, Acheampong JW, Appiah LT, Oparebea E, Akpalu A, Bedu-Addo G. The profile of risk factors and in-patient outcomes of stroke in Kumasi, Ghana. Ghana Med J. 2014;48(3):127-134. doi:10.4314/gmj.v48i3.2

10. Zewdie A, Debebe F, Kebede S, et al. Prospective assessment of patients with stroke in Tikur Anbessa Specialised Hospital, Addis Ababa, Ethiopia. African J Emerg Med. 2017:1-4. doi:10.1016/j. afjem.2017.11.001

11. Deljavan R, Farhoudi M, Sadeghi-Bazargani H. Stroke in-hospital survival and its predictors: the first results from Tabriz Stroke Registry of Iran. Int J Gen Med. 2018;11:233-240.

12. Fekadu G, Chelkeba L, Kebede A. Risk factors, clinical presentations and predictors of stroke among adult patients admitted to stroke unit of Jimma university medical center, south west Ethiopia: prospective observational study. BMC Neurol. 2019;19:1-11.

13. Walker RW, Wakefield K, Gray WK, Jusabani A, Swai M, Mugusi F. Case-fatality and disability in the Tanzanian Stroke Incidence Project cohort. Acta Neurol Scand. 2016;133(1):49-54. doi:10.1111/ ane. 12422

14. Walker RW, McLarty DG, Kitange HM, et al. Stroke mortality in urban and rural Tanzania. Lancet. 2000;355:1684-1687. doi:10.1016/ S0140-6736(00)02240-6
15. Abilleira S, Lucente G, Ribera A, Permanyer-Miralda G, Gallofré M. Patient-related features associated with a delay in seeking care after stroke. Eur $J$ Neurol. 2011;18:850-856. doi:10.1111/j.14681331.2010.03258.x

16. Alemayehu CM, Birhanesilasie SK. Assessment of stoke patients: occurrence of unusually high number of haemorrhagic stroke cases in Tikur Anbessa specialized Hospital, Addis Ababa, Ethiopia. Clin Med Res. 2013;2(5):94-100. doi:10.11648/j.cmr.20130205.11

17. Misganaw A, Haregu TN, Deribe K, Tessema GA, Deribew A. National mortality burden due to communicable, non-communicable, and other diseases in Ethiopia, 1990 - 2015: findings from the Global Burden of Disease Study 2015. Popul Health Metr. 2017;15:29. doi:10.1186/s12963-017-0145-1

18. CDC. CDC in Ethiopia. Atlanta; 2019. Available from: www.cde. gov/global.

19. Sultan M, Debebe F, Azazh A, Hassen GW. Epidemiology of stroke patients in Tikur Anbessa Specialized Hospital: emphasizing clinical characteristics of Hemorrhagic Stroke Patients. Ethiop J Heal Dev. 2017;31(1):13-17.

20. Temesgen TG, Teshome B, Njogu P. Treatment outcomes and associated factors among hospitalized stroke patients at Shashemene Referral Hospital, Ethiopia. Stroke Res Treat. 2018;1-5.

21. Fekadu G, Adola B, Mosisa G, Shibiru T, Chelkeba L. Clinical characteristics and treatment outcomes among stroke patients hospitalized to Nekemte referral hospital, western Ethiopia. J Clin Neurosci. 2020;71:170-176. doi:10.1016/j.jocn.2019.08.075

22. CSA. Summary and statistical report of the 2007 population and housing census. Fed Democr Repub Ethiop Popul Census Comm. 2008;1-1084.

23. World Medical Association. Declaration of Helsinki: ethical principles for medical research involving human subjects. 2008:1-5. doi:10.1093/acprof:oso/9780199241323.003.0025

24. Stroke Foundation. Clinical guidelines for stroke management: summary of recommendations. 2017:1-61.

25. FMHACA. Standard treatment guidelines. 2014.

26. World Health Organization. WHO Monica Project: MONICA manual. Part IV: event registration. 1990. Available from: http://www.ktl. fi/publications/monica/manual/part4/iv-2.htm\#s1-1.

27. Berkowitz AL, Westover MB, Bianchi MT. Aspirin for acute stroke of unknown etiology in resource-limited settings: a decision analysis. Neurology. 2014;83:787-793. doi:10.1212/WNL.0000000000000730

28. Gedefa B, Menna T, And TB, Abera H. Assessment of risk factors and treatment outcome of stroke admissions at St. Paul's Teaching Hospital, Addis Ababa, Ethiopia. J Neurol Neurophysiol. 2017;8 (3):431. doi:10.4172/2155-9562.1000431

29. Erkabu SG, Agedie Y, Mihretu DD, Semere A, Alemu YM. Ischemic and hemorrhagic stroke in Bahir Dar, Ethiopia: a retrospective hospital-based study. J Stroke Cerebrovasc Dis. 2018;27(6):1533-1538. doi:10.1016/j.jstrokecerebrovasdis.2017.12.050

30. Shenkutie Greffie E. Risk factors, clinical pattern and outcome of stroke in a Referral Hospital, Northwest Ethiopia. Clin Med Res. 2015;4(6):182. doi:10.11648/j.cmr.20150406.13

31. Gufue ZH, Gizaw NF, Ayele W, et al. Survival of stroke patients according to hypertension status in Northern Ethiopia: seven years retrospective cohort study. Vasc Health Risk Manag. 2020;16:389401.

32. Baye M, Hintze A, Gordon-murer C, Mariscal T. Stroke characteristics and outcomes of adult patients in Northwest Ethiopia. Front Neurol. 2020;11:428. doi:10.3389/fneur.2020.00428

33. Deresse B, Shaweno D. Epidemiology and in-hospital outcome of stroke in South Ethiopia. J Neurol Sci. 2015;355(1-2):138-142. doi:10.1016/j.jns.2015.06.001

34. Alene M, Assemie MA, Yismaw L, Ketema DB. Magnitude of risk factors and in-hospital mortality of stroke in Ethiopia: a systematic review and meta-analysis. BMC Neurol. 2020;20:309. 
35. Garbusinski JM, Van Der Sande MAB, Bartholome EJ, et al. Stroke presentation and outcome in developing countries: a prospective study in the Gambia. Stroke. 2005;36:1388-1393. doi:10.1161/01. STR.0000170717.91591.7d

36. Nkoke C, Lekoubou A, Balti E, Kengne AP. Stroke mortality and its determinants in a resource-limited setting: a prospective cohort study in Yaounde, Cameroon. J Neurol Sci. 2015;358(1-2):113-117. doi:10.1016/j.jns.2015.08.033

37. Hennekens $\mathrm{CH}$, Schneider WR. The need for wider and appropriate utilization of aspirin and statins in the treatment and prevention of cardiovascular disease. Expert Rev Cardiovasc Ther. 2008;6(1):95107. doi:10.1586/14779072.6.1.95

38. Kaduka L, Muniu E, Oduor C, et al. Stroke mortality in Kenya ' s public tertiary hospitals: a prospective facility-based study. Cerebrovasc Dis Extra. 2018;9:70-79. doi:10.1159/000488205

39. Desita Z, Zewdu W. CT scan patterns of stroke at the University of Gondar Hospital, North West Ethiopia. Br J Med Med Res. 2015;6 (9):882-888. doi:10.9734/bjmmr/2015/14849

40. Gebreyohannes EA, Bhagavathula AS, Abebe TB, Seid MA, Haile KT. In-hospital mortality among ischemic stroke patients in Gondar University Hospital: a retrospective cohort study. Stroke Res Treat. 2019;2019:7. doi:10.1155/2019/7275063
41. Hundt GL, Stuttaford M, Ngoma B. The social diagnostics of strokelike symptoms: healers, doctors and prophets in agincourt, limpopo province, South Africa. J Biosoc Sci. 2004;36(4):433-443. doi:10.1017/S0021932004006662

42. Mekonen HH, Birhanu MM, MossieID TB, Gebreslassie HT. Factors associated with stroke among adult patients with hypertension in Ayder comprehensive specialized Hospital, Tigray, Ethiopia, 2018: a case-control study. PLoS One. 2020;15(2):e0228650. doi:10.1371/ journal.pone. 0228650

43. Smith EE, Shobha N, Dai D, et al. Risk score for in-hospital ischemic stroke mortality derived and validated within the get with the guidelines - stroke program. Circulation. 2010;122:1496-1504. doi:10.1161/CIRCULATIONAHA.109.932822

44. Nedeltchev K, Renz N, Karameshev A, et al. Predictors of early mortality after acute ischaemic stroke. Swiss Med Wkly. 2010;140 (17-18):254-259.
Vascular Health and Risk Management

\section{Publish your work in this journal}

Vascular Health and Risk Management is an international, peerreviewed journal of therapeutics and risk management, focusing on concise rapid reporting of clinical studies on the processes involved in the maintenance of vascular health; the monitoring, prevention and treatment of vascular disease and its sequelae; and the involvement

\section{Dovepress}

of metabolic disorders, particularly diabetes. This journal is indexed on PubMed Central and MedLine. The manuscript management system is completely online and includes a very quick and fair peerreview system, which is all easy to use. Visit http://www.dovepress. com/testimonials.php to read real quotes from published authors. 